\title{
Novel RFID and Ontology based Home Localization System for Misplaced Objects
}

\author{
Son Minh Huynh, David Parry, Member, IEEE, A. C. M. Fong, Senior Member, IEEE, \\ and Jie Tang, Senior Member, IEEE
}

\begin{abstract}
Smart Home systems that can help track and localize objects within the home have been an important part of Consumer Electronics research in recent years. In practical terms, these systems can help people find certain things, such as glasses, wallets, keys, etc., and it is often troublesome to look for them. Previous research has shown great achievements in terms of localization accuracy of up to about $10 \mathrm{~cm}$. However, no existing solution gives a meaningful description of the location of the misplaced object related to important known areas in the home or other space, which are easily understood by the user. This study proposes an RFID and ontology-based solution to localize the easily-lost objects based on three nearest reference points and recommends how to build the localization system so that it can be easily integrated into the entire Smart Home system that adheres to the Semantic Web protocols. The solution generates localization results as user-friendly statements, e.g. "The glasses are at the kitchen table". The current highest accuracy rate of $87.5 \%$ encourages enhancement of the technique and implementation of the complete system ${ }^{l}$.
\end{abstract}

Index Terms - Smart Home, assisted living, lost object search, localization, RFID, Semantic Web, Internet of Things, ontology.

\section{INTRODUCTION}

Smart Home ( $\mathrm{SH})$ systems assist users with daily activities within the home by applying ubiquitous computing. Implementation of ubiquitous computing infrastructure helps manage objects in the home and enable real-world searches, which differ from the virtual world search of current web search engines. The Global Positioning System (GPS) is considered as the best technology for outdoor localization systems but performs poorly in accuracy and precision in the indoor environment [1]. In addition, satellite signals received in the indoor environment are weak and therefore GPS is unlikely to be able to be used indoor [2], [3]. To overcome these difficulties, various wireless technologies have been

${ }^{1}$ S. M. Huynh, D. Parry, and A. C. M. Fong are with the School of Computing and Mathematical Sciences, Auckland University of Technology, Auckland, New Zealand (e-mail: steve.huynh@aut.ac.nz, dave.parry@aut.ac.nz, alvis.fong@aut.ac.nz).

J. Tang is with the Department of Computer Science and Technology, Tsinghua University, Beijing, China (e-mail: jietang@tsinghua.edu.cn).

Contributed Paper

Original manuscript received 05/28/14

Revised manuscript received 06/16/14

Current version published 09/23/14

Electronic version published 09/23/14. experimented with including infrared, bluetooth, IEEE 802.11 wireless LAN, ultrasonic, video camera, and Radio Frequency IDentification (RFID) [1], [4], [5]. RFID seems to be the most suitable candidate for tracking and localization in SH systems because of its robustness, low price, and flexibility [5]. In addition, RFID can uniquely identify physical artifacts and can be used as a universal entry point to integrate them into the Internet of Things (IOTs) [6], and thus, RFID presents a viable solution in building $\mathrm{SH}$ systems. Considered as a main application domain of IOTs, SH systems have attracted much attention recently, such as the study by Song et al. [7].

An RFID system has two main components, transponders (RFID tags) and detectors (RFID readers). RFID tags are divided into three categories, active tags, passive tags, and semi-passive tags. The type of RFID system is based on whether the tags are active, passive or semi-passive. For example, if the system uses only active tags then it is called an active RFID system. An active tag has its own power source (battery) to run its internal circuitry and to broadcast signals to readers. A passive tag does not have its own power source and cannot initiate communication with the reader. It is activated by and draws energy from the reader's signal to send back signals to the reader. A semi-passive tag does not initiate the communication with the reader either, but it has its own battery to run its internal circuitry. Active tags are bigger in size, transmit longer-range signals, and are more expensive than passive tags.

This paper proposes a solution for localization of easily-lost objects (LOs) within the home and describes a possible implementation of the whole system. The solution uses passive RFID technology for localization and stores information as an ontology. Information stored as an ontology enables the application of reasoning to the search and facilitates its integration with the entire SH system [8]. It also ensures adaptability with the emerging Semantic Web. Prior to this study, many researchers have proposed various RFID solutions for indoor localization. However, these solutions tend to be confined to the prototype or laboratory experimentation stage and much remains to be done to bring them into everyday application due to issues with cost, accuracy, and usability [9]. Unlike these existing solutions, this research presents a solution that is novel and viable to be deployed on a large-scale in consumer home environments. 
The solution in this study can give the location of the LO with enough detail to show it is on/in (at) a piece of furniture or between some pieces of furniture in the room, for example, "the glasses are at the kitchen table". The fact that the LO is either on/in a piece of furniture or between some pieces of furniture in the room means localization of the LO is classified into two types. Localization of the LO presents on/in a piece of furniture is classified as localization Type 1 . On the other hand, localization Type 2 is the localization of the LO between pieces of furniture.

This paper is a significantly expanded version of the authors' conference paper [10]. It adds an overview of existing indoor localization technologies and analyzes current RFID-based solutions to stand-out the contribution of the solution of this research. In addition, it provides a design of the whole system including hardware, software, searching scenarios and ontologies queries. It also augments the Implementation and Experiments section and substantially extends the Results and Discussion section to reinforce the appropriateness and the feasibility of the proposed solution.

The rest of this paper is organized as follows. First, Section II analyzes related work to identify challenges with indoor localization and to find out the role RFID can play. Then, Section III describes the solution of this study and Section IV explains the implementation and experiments. Further, Section Error! Reference source not found. presents results and discussion of the proposed solution. Finally, Section VI concludes the paper.

\section{Literature ANALYSIS}

\section{A. Challenges with indoor localization}

Many studies have emphasized the development of a robust system for indoor localization [9]. However, they all face difficulties in developing a real-world system and thus are still in prototype or experimental stages. Unlike outdoor environments, where GPS has been found effective in localization with an accuracy obtainable of approximately 3 $\mathrm{m}$, indoor environments typically demand more in accuracy of a localization system if the system is to be useful [2], [3]. Combining these two studies [2], [3] with another study by Fortin-Simard et al. [5], the following six challenges to an indoor localization system have been derived.

- Accuracy: Indoor environments are limited by the sizes of the rooms and buildings. Indoor objects are relatively small and close to each other. Thus, the accuracy of the system should be high, within 3 feet (about $1 \mathrm{~m}$ ) [9], [11].

- No need for direct line-of-sight: there are many objects within an indoor environment and therefore these objects can stand in between the detector and the tracking object. A system that needs direct line-of-sight is not suitable.
- Low cost: Due to the number of tracking objects, rooms, and buildings. The cost always needs to be considered to see whether implementing the system is viable for widespread consumer SH applications.

- Flexibility: The system should be flexible enough to be applied to different tracking items, different rooms and buildings with minimum modification.

- Non-intrusiveness: the localization system should assist home users, not intrude on their everyday lives. For example, a camera system is intrusive and not suitable for indoor tracking and localization for SH systems.

- Usability: The user should find it is easy to use the developed system. Many solutions present the localization result to the user as coordinates for the tracked object and it is hard for the user to interpret where it is in the room. For example, it is not at all easy for the user to quickly imagine the actual location of a tracked object having the coordinates $(3.8 \mathrm{~m}, 2.7 \mathrm{~m})$ in a room. The user would find it much easier if the system tells them that the object is at the kitchen table, for example.

\section{B. RFID in indoor localization}

RFID has been the focus of research for a number of years and has been successfully applied in a number of scientific and technical fields, such as medicine, engineering, the aeronautics industry supply chain, and the retail industry [12]. In recent years, much attention has been paid to using RFID in indoor tracking and localization. Benefits of using RFID include; no need for direct line-of-sight, low cost, flexibility, and non-intrusiveness [4], [5].

Many studies have proposed RFID solutions for indoor localization. TABLE I shows some of these studies from the last decade. The table gives a summary of each proposed solution with the technology (active, passive, or semi-passive RFID) and its accuracy. The analysis of these studies is summarized in TABLE II that lists the challenges the solutions meet as well as specifying their limitations. It can be seen that no solution has met all six identified challenges mentioned in Section A. "Usability", especially, has not been satisfied by any study, though passive RFID technology solutions have already satisfied the "Accuracy", "No need direct line-of-sight", "Low cost", "Flexibility", and "Nonintrusiveness" requirements. Most of the existing solutions present the localization results as coordinates of the tracking objects which is not user-friendly. Only the solution by Tesoriero et al. [1] developed a virtual map showing the position of the tracking object. However, the user still needs to relate the virtual map with the actual room and identify the physical location of the tracking object from its position on the virtual map. In the next section, this study presents a new solution that addresses the limitations of the existing literature, focusing especially on "Usability". 
TABLE I

RFID BASED SOLUTIONS

\begin{tabular}{cll}
\hline \hline Study & Technology & \multicolumn{1}{c}{ Solution } \\
\hline Ni et al., 2004 [4] Active RFID & $\begin{array}{l}\text { This is a 2D solution that uses reference tags. The distance between an active RFID tag and the RFID } \\
\text { reader is estimated based on the power level }(1-8) \text { of the tag detected. The position of the tracking tag } \\
\text { is decided from its } \mathrm{k} \text { nearest neighbors (k nearest reference tags) and the highest accuracy is achieved } \\
\text { when } \mathrm{k}=4 . \text { To improve accuracy, multiple readers are used with the optimal number of readers being } \\
\mathrm{n}=4 . \text { The localization error of the system is less than 2 m. }\end{array}$
\end{tabular}

Tesoriero et al., $2009 \quad$ Passive RFID

[1]

Almaaitah et al., 2010

[13]

Saad and Nakad, 2011

[14]

Yuhong and Ya, 2012

[15]

Fortin-Simard et al., 2012 [5]

Brchan et al., 2012

[16]

DiGiampaolo and

Martinelli, 2012 [17]

Han et al., 2012 [18] Active RFID

Athalye et al., 2013

[19]

Semi-passive RFID

Passive RFID

Passive RFID

Passive RFID

Active RFID

Passive RFID
This is a 2D solution that divides the floor surface into a grid of small squared location units. Each physical location unit is tagged by a passive RFID tag and mapped to a position on the virtual map in the system. The tracking object is attached with an RFID reader. Based on which tag the reader is reading, the tracking object's location is mapped and shown on the virtual map in the system. The localization error of the system is about $0.9 \mathrm{~m}$.

Passive RFID This paper introduces two methods for 3D localization of a passive RFID tag. The first method named Adaptive Power Multilateration (APM) uses 4 RFID readers and localizes the tag based on the minimal interrogation power and multilateration. The second method named Adaptive Power with Antenna Array (APAA) uses a single RFID reader equipped with horizontal and vertical smart antennas and the reader's adaptive power levels. The APM method gives an accuracy of $0.32 \mathrm{~m}$ while the APAA method gives the accuracy of $0.48 \mathrm{~m}$.

This 2D solution also attaches the reader to the tracking object. The passive RFID tags are placed along the object path as reference tags. From the distances between the reader and the reference tags calculated, the position of the object is derived. To have high accuracy, both RSSI and angledependent loss factor are involved in the proposed algorithm using Kalman filter. Even not specified, this can be applied to both $2 \mathrm{D}$ and $3 \mathrm{D}$ environment setting. The localization error of the system is about $0.1 \mathrm{~m}$.

This is a $2 \mathrm{D}$ solution that calculates the coordinates of the tracked passive RFID tag based on Angle of Arrival (AOA) and Time Difference of Arrival (TDOA) rather than RSSI. The system employs two RFID readers whirling around their fixed axes to scan tags in the room. The localization error of the system is less than $1 \mathrm{~m}$.

This is a 2D solution that proposes a new algorithmic approach to localize the tracked passive RFID tag based on elliptical trilateration and fuzzy logic. Though the solution uses only RSSI as the input parameter to the algorithm, it employs multiple filters (iteration based filter, Gaussian mean weighting filter, delta filter and multi-point location filter) to achieve high accuracy. The localization error of the system is about $0.14 \mathrm{~m}$.

This is a 2D solution with an extension to a 3D solution that localizes the tracked active RFID tag base on RSSI with the use of multiple propagation models to improve the accuracy of the system. The system uses reference tags and aims to improve the LANDMARC system. However, the localization error of the 2D model does not show improvement, $58.3 \%$ of the time at less than $1 \mathrm{~m}$ and $1.7 \%$ of the time at more than 2 $\mathrm{m}$. In the 3D model, the error is $9.7 \%$ of the time at more than $3 \mathrm{~m}$.

In this solution (2D solution), passive reference tags are placed on the ceiling and the tracking object is attached with the RFID reader. A Quantized Extended Kalman Filter is applied in the algorithm for distance measurements. The localization error of the system is about $0.1 \mathrm{~m}$.

This is a 3D localization solution based on active reference tags placed as a 3D grid. The distance between 2 adjacent tags is $1 \mathrm{~m}$. Each reader has 2 antennas. The algorithm uses 2 RSSI values reported by the 2 antennas and applies filter rules to estimate the distance between the tag and the reader. The average localization error of the system is about $0.54 \mathrm{~m}$.

This is both a $2 \mathrm{D}$ and $3 \mathrm{D}$ solution which uses passive RFID tags as reference tags and a newly developed semi-passive RFID tag component called sensatag for tagging the tracked object. The sensatag has dual functionality, first, detecting and decoding backscatter signals from the passive RFID tags, and second, communicating with the reader just like a standard passive tag. The system's average error is about $0.30 \mathrm{~m}$. This solution is applied to find the exact placement of items on shelves. 
TABLE II

RFID BASED SOLUTIONS ANALYSIS

Study Challenges meet $\quad$ Limitations

Ni et al., 2004 No need for direct line-of-sight, - Huge amount of RFID tags need to be placed on the floor.

[4]

flexibility, and non-intrusiveness - Expensive due to the use of so many active tags

- Effort in maintaining the active RFID tags' batteries

- The reference tags make sense to computers but are hard for humans to interpret as physical locations.

Tesoriero et Accuracy, no need for direct - Huge amount of RFID tags need to be placed on the floor.

al., 2009 [1] line-of-sight, and non- - Expensive due to each tracking object being attached with an RFID reader.

intrusiveness - Hard or impossible to attach the RFID reader to small objects, such as keys, glasses, wallets etc.

Almaaitah et Accuracy, no need for direct - The computed coordinates make sense to computers but are hard for humans to interpret as al., 2010 [13] line-of-sight, low cost, physical locations.

flexibility, and non-intrusiveness

Saad and

Nakad, 2011

Accuracy, no need for direct

[14]

line-of-sight, low cost, and non-

intrusiveness

- Not flexible because the objects need to have fixed routes.

- Hard or impossible to attach the RFID reader to small objects, such as keys, glasses, wallets etc.

Yuhong and Ya, 2012 [15]

Fortin-Simard et al., 2012 [5]

Brchan et al., 2012 [16]

DiGiampaolo and Martinelli, 2012 [17]

Han et al., 2012 [18]
Accuracy, no need for direct line-of-sight, low cost, and flexibility
- The motors required for whirling the RFID readers can generate noise and can be annoying. - The computed coordinates make sense to computers but are hard for humans to interpret as physical locations.

Accuracy, no need for direct - Does not test the solution with various environmental settings, such as different sizes and shapes line-of-sight, low cost, of tracked objects, relocating furniture in the room etc.

flexibility, and non-intrusiveness - The computed coordinates make sense to computers but are hard for humans to interpret as physical locations.

No need for direct line-of-sight, flexibility, and non-intrusiveness

- Expensive due to the use of active tags

- Effort to maintain the active RFID tags' batteries

- The reference tags make sense to computers but are hard for humans to interpret as physical locations.

Accuracy, no need for direct

- Hard or impossible to attach the RFID reader to small objects, such as keys, glasses, wallets etc.

- The accuracy would decrease when the tracked object moves to higher ceiling rooms.

- The computed coordinates make sense to computers but are hard for humans to interpret as physical locations.

intrusiveness

Accuracy, no need for direct - Expensive due to the use of a huge amount of active tags
line-of-sight, and non- $\quad$ - Effort to maintain the active RFID tags' batteries

intrusiveness - Not practical and perhaps hazardous to setup a 3D grid of reference tags in the room.

- The reference tags make sense to computers but are hard for humans to interpret as physical locations.

Athalye et al., Accuracy, no need for direct - Effort to maintain the semi-passive RFID tags' batteries

2013 [19] line-of-sight, flexibility, and non-intrusiveness

\section{Solution}

\section{A. System Design}

Fig. 1 shows an overview of the Home Localization System for Misplaced objects (HLSM). HLSM consists of a central server, fixed RFID readers (D1-4), and mobile RFID readers (e.g. RFID reader smart phones). The fixed readers connect to the central server either by Ethernet or WiFi depending on the reader type, and the mobile readers communicate with the server via WiFi.

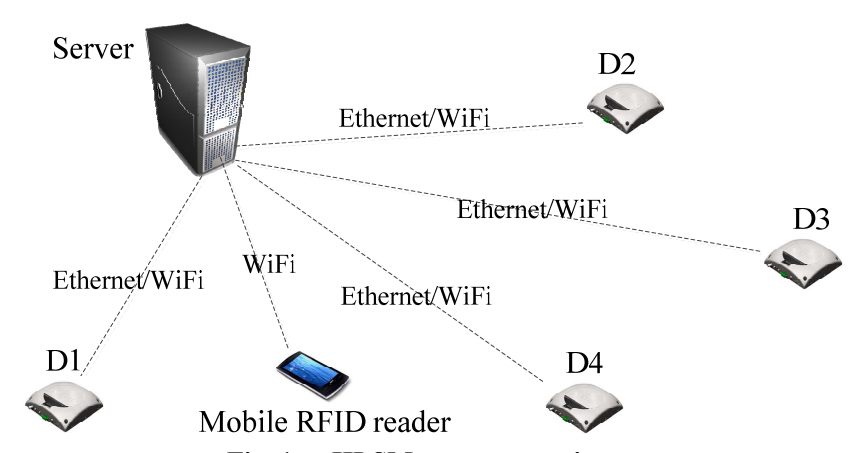

Fig. 1. HLSM system overview. 
Fig. 2 illustrates the software architecture of HLSM. The central server consists of a Web Server, an Application Server, and an Ontology Database. Mobile RFID readers (smart phones) communicate with the Web Server and the fixed RFID readers communicate with the Application Server. The Application Server and the Web Server query the Ontology Database using SPARQL (SPARQL Protocol and RDF Query Language), an RDF query language. RDF (Resource Description Framework) is a language for describing information and resources in Semantic Web environments.

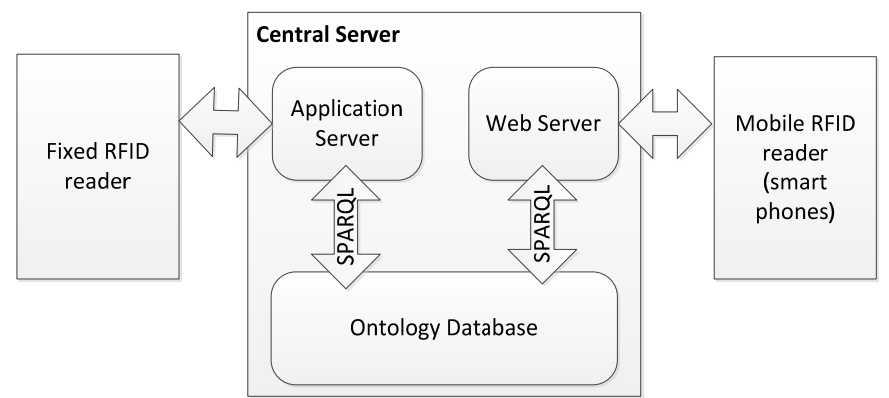

Fig. 2. HLSM software architecture overview.

\section{B. Tag placing strategy and Localization Rule}

Fig. 3 describes the RFID reader and tag placing strategy in the proposed solution. D1-4 are fixed RFID readers used as high-level navigation landmarks (HLNLs) placed at room entry doors. T1-8 are passive RFID tags used as detailed navigation landmarks (DTNLs), which serve as reference points. Each piece of furniture where the passive RFID tagged LO is possibly located, e.g. kitchen table, is tagged with four DTNLs, one at each corner. The HLNLs help identify in which room the searched for object could possibly be, while the DTNLs help narrow it down to which part of the room the object is located. For the detailed search, the LO is localized from its three nearest identified DTNLs. The system, based on the below Localization Rule, then generates the localization result from these three DTNLs.

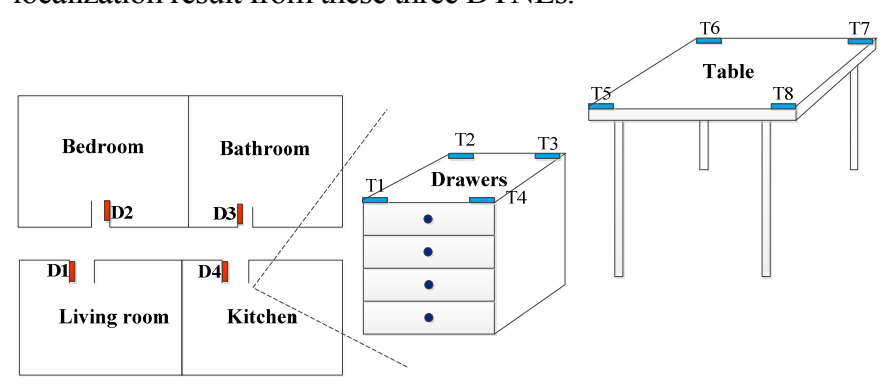

Fig. 3. RFID tag placements.

Localization Rule: If any two of the three nearest DTNLs identified by HLSM belong to the same piece of furniture then it is concluded that the LO is "at" (on/in) that piece of furniture. Otherwise, it is said that the LO is between the pieces of furniture that the three nearest DTNLs belong to.

The reason for choosing three as the number of nearest DTNLs $(n)$ and two as the threshold $(m)$ was based on the experimental results of Section V. Different sets of $(n, m)$ were experimented on to finalize the optimal set $(n=3, m=2)$ for the Localization Rule.

\section{Search Scenarios}

The HLSM Web Server retrieves data from and saves data to the Ontology Database, which contains the LO Ontology. The LO Ontology stores the last HLNL for the LO. When the $\mathrm{LO}$ is moved to another room, the fixed RFID reader at the room entry door detects and sends the RFID tag information of the LO to the Application Server to update the HLNL of the LO in the LO Ontology. Fig. 4 illustrates this scenario.

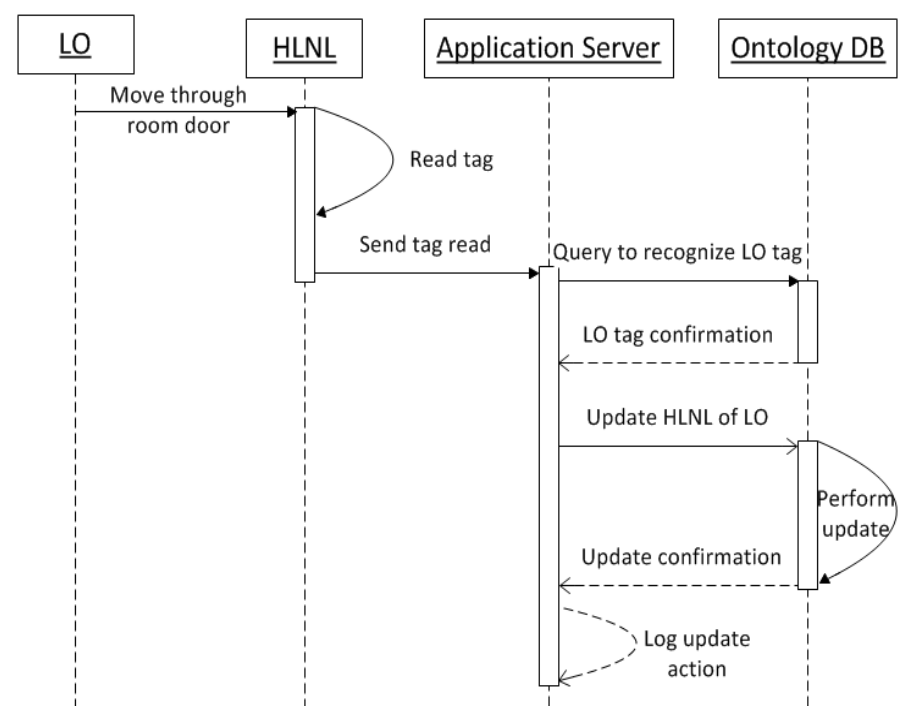

Fig. 4. LO moves to another room.

When the home user wants to search for an LO, he/she carries an RFID reader smart phone and accesses the HLSM Web application via the developed application on the phone. The user can interact with the Web application either via the Web GUI or an interactive voice dialog system (e.g. voiceXML). Once the Web Server receives a request to search for the LO, it accesses the Ontology and returns information about the room the LO is currently located based on the last HLNL associated with the LO. The user goes to that room and starts the detailed search. At this stage, an Avalanche search can be applied. An example of an Avalanche search is; if the reading range of the mobile RFID is, for example, $1 \mathrm{~m}$, then the user can choose a point at the room's center as center point $C$ and keep rotating around this point with an increasing radius; $1 \mathrm{~m}, 3 \mathrm{~m}, 5 \mathrm{~m}$ and so forth until the LO is recognized. Choosing the radii of $1(m)+1(m) \times 2 n(n \in \mathbb{V})$, with $n$ starting from 0 and keeps increasing, makes sure that all parts of the room are covered in the search. Once the LO is recognized, DTNLs are read and sent to the Web Server by the phone to identify the three nearest DTNLs of the LO. The Web Server then applies the Localization Rule aforementioned to generate the localization result and returns the result to the phone. The user uses this generated result to find the LO. Once the LO is found, its actual location is sent back to the server to update the history dataset where data mining technique may be applied. Figs. 5 and 6 show these two scenarios. 


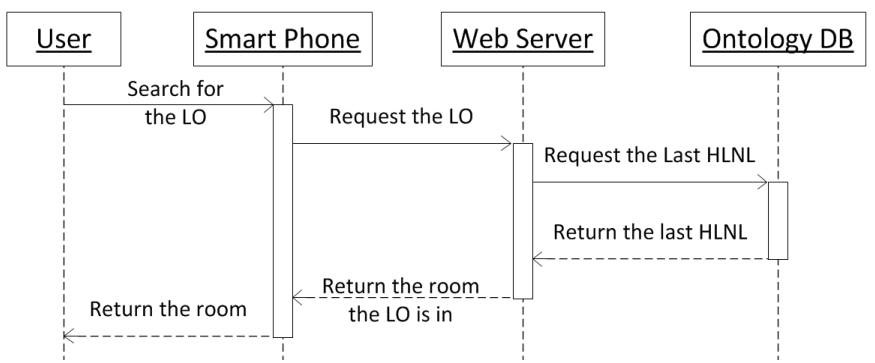

Fig. 5. High level search.

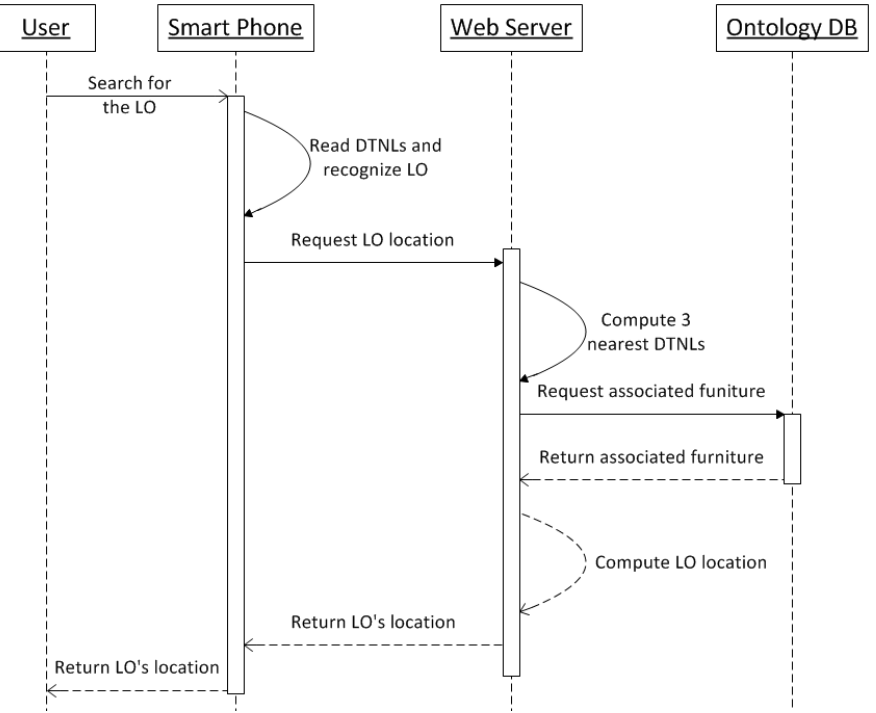

Fig. 6. Detailed search.

\section{LO Ontology Design}

Fig. 7 shows the LO ontology (simplified). Five classes are "LO", "Landmark", "Furniture", "Room", and "User". The object properties, "isIn", "isAt", "isBetween", "belongsTo", "isOwnedBy", "isNear1", "isNear2", "isNear3", describe the relationships among these classes. "isIn" connects "LO" and "Room". "isAt" and "isBetween" respectively describe the "LO" being at or between "Furniture". "belongsTo" indicates that "Landmark" belongs to "Furniture". "isOwnedBy" connects "LO" and "User". "isNear1" indicates the nearest "Landmark" of the "LO". "isNear2" connects the "LO" to the 2nd nearest "Landmark" and so forth. The individuals of the classes are represented as diamonds in the diagram.

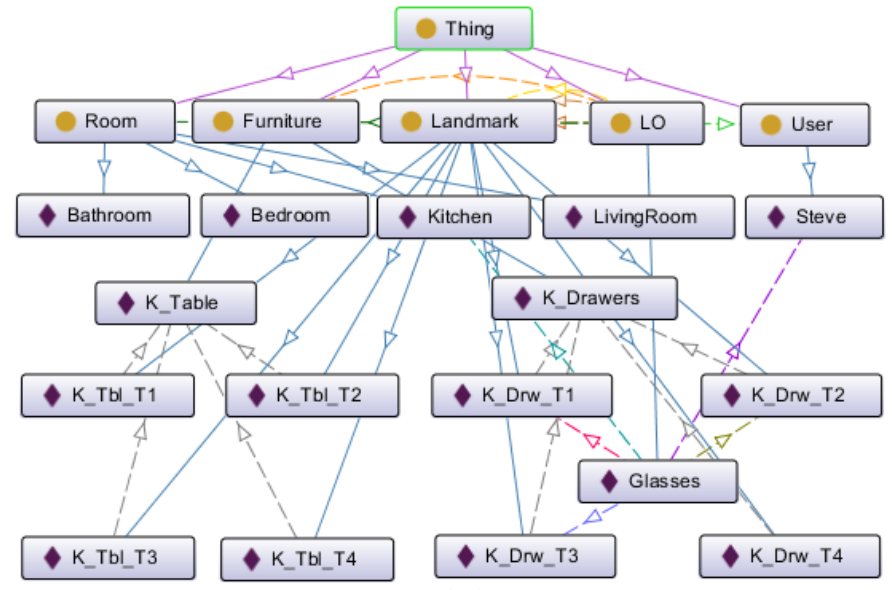

Fig. 7. LO Ontology.
As this is a simplified version, information is stylized and some details are not mentioned. For example, the HLNLs are not presented. Instead, the LO (glasses) is directly connected to the room (Kitchen). The completed LO ontology would have many more classes and individuals. Nevertheless, this simplified version of the LO ontology is enough to illustrate how the LO ontology looks as well as providing enough information to support ontology queries for this study. The LO ontology was deployed on a Jena Fuseki server version 1.0.0. Below are some sample SPARQL queries for querying the LO ontology and query results in JSON (JavaScript Object Notation) format. In these sample queries, "lo_ontology_iri" is an ontology IRI (Internationalized Resource Identifier) that is used to identify the LO ontology [23].

Query 1: Find which room the Glasses are in.

PREFIX lo: <lo_ontology_iri\#>

SELECT * ${ }^{*}$ lo:Glasses lo:isln ?z $\}$

Query 1's result: the answer is Kitchen (the bold text in the JSON result script below).

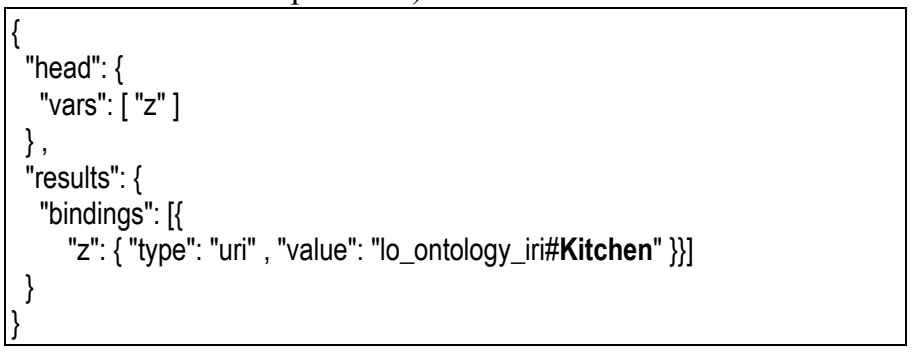

Query 2: Find the Glasses' nearest DTNL.

PREFIX I0: <lo_ontology_irit\#>

SELECT * ${ }^{*}$ \{lo:Glasses lo:isNear1 ?z $\}$

Query 2's result: the answer is K_Drw_T1 (the bold text in the JSON result script below).

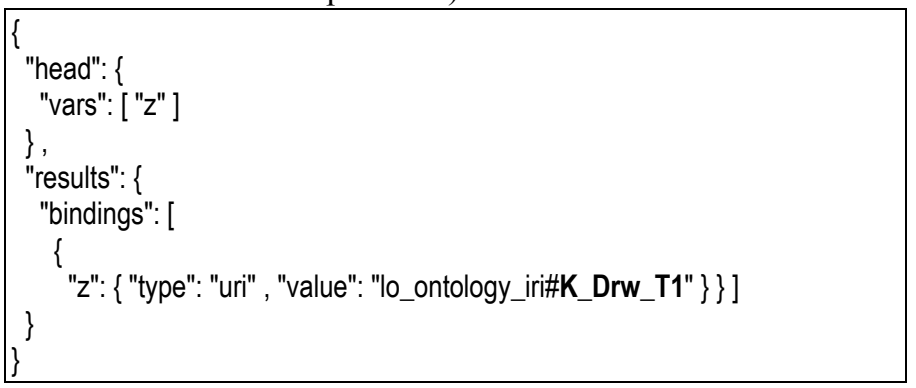

Query 3: Update the nearest DTNL of the Glasses from K_Drw_T1 to K_Tbl_T4. This update consists of 2 steps. The first step is deleting the existing RDF statement $<$ Glasses $><$ isNear $1><K_{-}$Drw_Tl $>$. The second step is inserting the new $\mathrm{RDF}$ statement $<$ Glasses $><$ isNear $1><K \_T b l \_T 4>$. PREFIX Io: <lo_ontology_irit\#> DELETE DATA $\{10: G l a s s e s$ lo:isNear1 lo:K_Drw_T1\}; INSERT DATA \{lo:Glasses lo:isNear1 lo:K_Tbl_T4\}; 


\section{IMPLEMENTATION AND EXPERIMENTS}

\section{A. Implementation}

The System Design part in Section III.A for the Web Server and the Application Server is technology independent. They can be implemented using any suitable technology, such as Java, .Net or PHP. In this research, Java technology was used to develop the HLSM system. The Web Server is developed using JSP technology and the Jena ontology API to query the LO ontology. The Application Server is written in Java and also uses the Jena ontology API. Jena Fuseki is used to host the LO ontology.

To experiment and test HLSM, focusing on the detailed search, which is the main part of the HLSM system, the authors have developed a Java application prototype to read and process data received from the mobile reader. The mobile reader keeps sending passive RFID tag information it reads to the Java application prototype. The Java application prototype checks all these tags to find the LO. Once the LO is recognized, the Java application prototype identifies the three nearest DTNLs to the LO based on signal strength (RSSI). Finally, the Java application prototype, which implements the Localization Rule, determines whether the LO is at (on/in) a specific piece of furniture or between some pieces of furniture.

\section{B. Experiments}

Fig. 8 shows the experimental setup. 24 UHF RFID tags were placed as DTNLs on 6 pieces of furniture (f) in the experiment room and 1 UHF RFID tag was placed as the LO. The sizes of the tables were $45 \mathrm{~cm} \times 45 \mathrm{~cm}, 110 \mathrm{~cm} \times 75 \mathrm{~cm}$, and $160 \mathrm{~cm} \times 80 \mathrm{~cm}$. The distances between any two pieces of furniture were from $170 \mathrm{~cm}$ to $250 \mathrm{~cm}$ to guarantee that when the LO was placed on any piece of furniture, its four actual nearest DTNLs were the four tags on that piece of furniture.

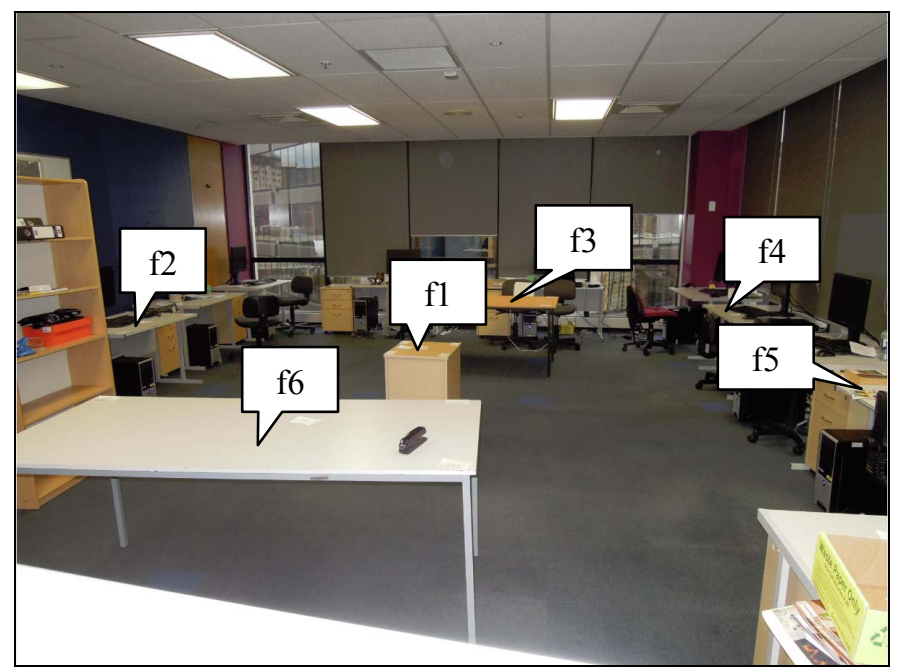

Fig. 8. Experimental setup.

Experiments were conducted on different combinations of the number of nearest DTNLs $(n)$ and the threshold $(m)$ to find out the optimal set of $(n, m)$ to form the Localization Rule in Section III.B. $m$ should be greater than $n / 2$ and less than or equal to 4 , which is the number of DTNLs placed on a piece of furniture. In total, 7 sets of $(n, m)$ were experimented on. They were $(3,2),(3,3),(4,3),(4,4),(5,3),(5,4)$, and $(6,4)$. Results generated from the Java application prototype for each combination of $(n, m)$ were recorded and compared to determine the optimal combination (TABLE III).

In total, 100 test cases were conducted to formulate the Localization Rule (Section III.B) as well as to obtain the localization accuracy of the HLSM system. A UHF Gen2 RFID tag reader was used as a mobile reader. The RFID antenna was pointed and moved towards the LO until the Java application prototype gave out the results. For localization Type 1 (LO isAt PieceOfFurniture), the LO was placed on three differently sized furniture types, five different positions on each piece of furniture and the antenna was approached from different directions for each position. 80 test cases in total were conducted for this localization type. In these 80 test cases, 40 test cases were for $45 \mathrm{~cm} \times 45 \mathrm{~cm}$ furniture, 20 test cases were for $110 \mathrm{~cm} \times 75 \mathrm{~cm}$ furniture, and 20 test cases were for $160 \mathrm{~cm} \times 80 \mathrm{~cm}$ furniture. For localization Type 2 , LO is between furniture, the LO was placed in between 2, 3, and 4 pieces of furniture and 20 test cases in total were conducted.

\section{RESUlts AND Discussion}

TABLE III shows the experimental results. The successful localization rate reaches $87.5 \%$ when the $\mathrm{LO}$ is at the furniture (Type 1). The 3 nearest neighbor technique with a threshold of $2,(n=3, m=2)$, works the best for all three furniture sizes and hence is chosen for the proposed solution. The smaller the furniture, the better the accuracy. This suggests placements of more than four reference tags on larger sized furniture would increase localization accuracy. Though accuracy for localization Type 2 is low (10\%), the localization result still narrows down the location of the LO. For example, the actual location was between $\mathrm{f} 3$ and $\mathrm{f} 4$, and the program gave a result between $\mathrm{f} 3, \mathrm{f} 4$ and $\mathrm{f} 5$, which helped in the search of the LO. In addition, it should be pointed out that localization Type 1 is more important than localization Type 2 and therefore should be given priority. Usually, objects are put in or on furniture ("is at" or type 1) rather than on the floor ("is between" or type 2). The graph in Fig. 9 depicts the localization accuracy results of localization Type 1 for all three furniture sizes.

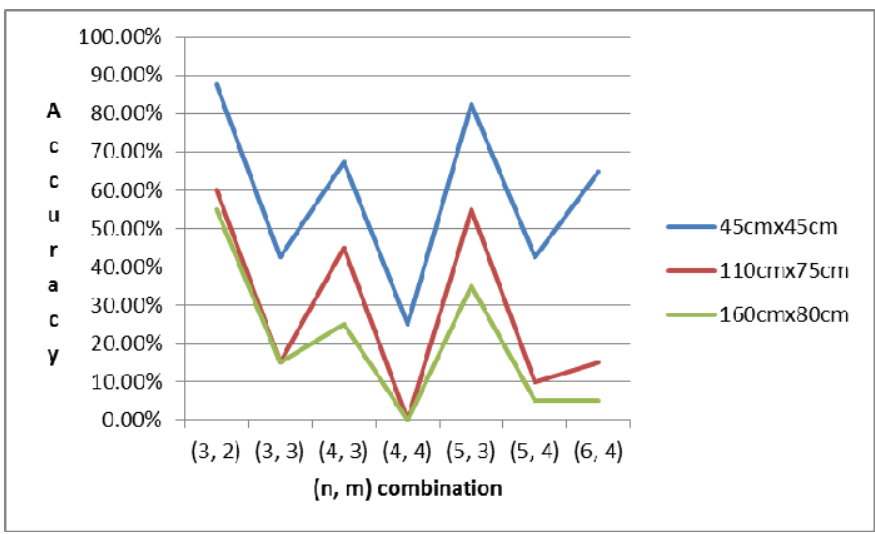

Fig. 9. Localization Type 1 accuracy results. 
TABLE II

EXPERIMENTATION RESULTS FOR HLSM SYSTEM PROTOTYPE

\begin{tabular}{|c|c|c|c|c|c|c|c|c|}
\hline Localization & Furniture & $\begin{array}{c}\text { Accuracy } \\
(n=3, m=2)\end{array}$ & $\begin{array}{c}\text { Accuracy } \\
(n=3, m=3)\end{array}$ & $\begin{array}{c}\text { Accuracy } \\
(n=4, m=3)\end{array}$ & $\begin{array}{c}\text { Accuracy } \\
(n=4, m=4)\end{array}$ & $\begin{array}{c}\text { Accuracy } \\
(n=5, m=3)\end{array}$ & $\begin{array}{c}\text { Accuracy } \\
(n=5, m=4)\end{array}$ & $\begin{array}{c}\text { Accuracy } \\
(n=6, m=4)\end{array}$ \\
\hline $\begin{array}{l}\text { Type } 1 \\
\text { (is at) }\end{array}$ & $\begin{array}{c}45 \mathrm{~cm} \times 45 \mathrm{~cm} \\
110 \mathrm{~cm} \times 75 \mathrm{~cm} \\
160 \mathrm{~cm} \times 80 \mathrm{~cm}\end{array}$ & $\begin{array}{c}35 / 40(87.5 \%) \\
12 / 20(60 \%) \\
11 / 20(55 \%)\end{array}$ & $\begin{array}{c}17 / 40(42.5 \%) \\
3 / 20(15 \%) \\
3 / 20(15 \%)\end{array}$ & $\begin{array}{c}27 / 40(67.5 \%) \\
9 / 20(45 \%) \\
5 / 20(25 \%)\end{array}$ & $\begin{array}{c}10 / 40(25 \%) \\
0 / 20(0 \%) \\
0 / 20(0 \%)\end{array}$ & $\begin{array}{c}33 / 40(82.5 \%) \\
11 / 20(55 \%) \\
7 / 20(35 \%)\end{array}$ & $\begin{array}{c}17 / 40(42.5 \%) \\
2 / 20(10 \%) \\
1 / 20(5 \%)\end{array}$ & $\begin{array}{c}26 / 40(65 \%) \\
3 / 20(15 \%) \\
1 / 20(5 \%)\end{array}$ \\
\hline $\begin{array}{c}\text { Type } 2 \\
\text { (is between) }\end{array}$ & & $2 / 20(10 \%)$ & $7 / 20(35 \%)$ & $7 / 20(35 \%)$ & $9 / 20(45 \%)$ & $3 / 20(15 \%)$ & $6 / 20(30 \%)$ & $3 / 20(15 \%)$ \\
\hline
\end{tabular}

In addition to less popularity of localization Type 2, the actual location of this type is sometimes itself at issue. The consequence is that the incorrect localization result might in fact be correct. Fig.10 illustrates this situation.

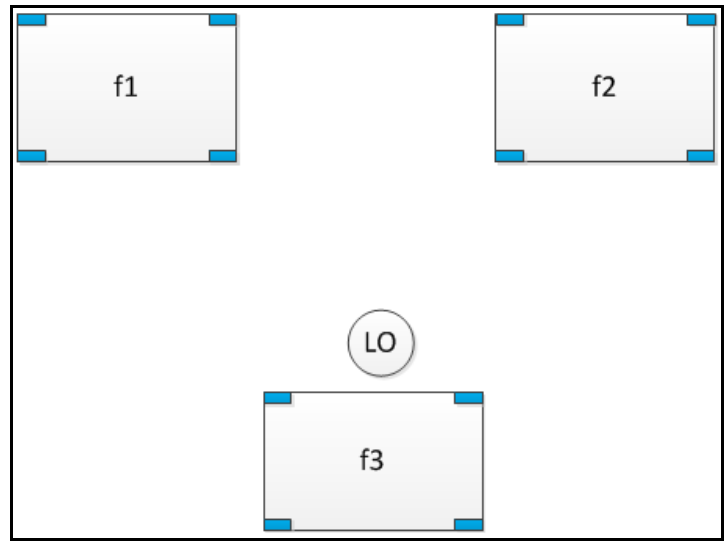

Fig. 10. Localization Type 2: disputed actual location.

It can be seen that, in this case, the actual location is stated to be "is between $\mathrm{f1}, \mathrm{f} 2$, and $\mathrm{f3}$ ". Therefore, the resulting location of "is at $\mathrm{f3}$ " is considered incorrect. However, this result is technically correct as the LO's four nearest DTNLs all belong to f3. From this example, the accuracy value of localization Type 2 does not really reflect how well the HLSM system performs.

TABLE IV summarizes the results of applying the performance metric defined based on the six challenges of indoor localization identified in Section II.A.

TABLE III

Performance Metric ApPlication Results For HLSM System PROTOTYPE

\begin{tabular}{lc}
\hline \multicolumn{1}{c}{ Challenge } & Result \\
\hline Accuracy & $87.5 \%$ \\
No need direct line-of-sight & OK \\
Low cost & OK \\
Flexibility & OK \\
Non-intrusiveness & OK \\
Usability & OK \\
\hline
\end{tabular}

In terms of accuracy, as explained above, the accuracy value of localization Type 1 should be used to measure the performance of the HLSM system rather than the localization Type 2 accuracy rate. In addition, though, there were three furniture sizes in the experiment and the larger sizes gave lower accuracy rates, the $87.5 \%$ accuracy rate coming from the smallest furniture size can be used as the accuracy rate of the HLSM system. The reason being that the larger sized furniture can be tagged with more than 4 RFID tags as can be seen from the experimentation results.

The other criteria constituting the performance metric also provide positive results. The fact that the HLSM system uses RFID technology means it does not need direct line-of-sight and it is non-intrusive [20]. As the system uses passive RFID tags which are cheap [21] and do not require batteries or maintenance and therefore have an indefinite operational life [20], the cost for building and operating the system should be low. Passive RFID tags are small in size and can be easily attached to different types and sizes of objects [20]. In addition, in the HLSM system, larger sized furniture can be tagged with more RFID tags than smaller sized furniture to achieve the same level of localization accuracy. These make the HLSM system flexible and being easily adapted to various home environment settings. In addition, although the system is named HLSM (Home Localization System for Misplaced objects), it can be used for many other indoor environments, such as hospitals and offices. Therefore, it can be called ILSM (Indoor Localization System of Misplaced objects). Finally, the system gives out the location in the form, the tracked object is at a specific piece of furniture or between pieces of furniture, e.g. "The glasses are at the kitchen table", which provides great usability for home users.

As HLSM is a web-based system, it is highly accessible to mobile RFID readers that can be smart phones or tablets. In addition, web-based system helps minimize fragmentation issue when developing software for mobile platforms. In HLSM, most of the functionalities are done on the Web Server, the mobile readers (smart phones/tablets) are mainly to read the RFID tags, pass the information to the Web Server and receive the result as HTML pages. As a result of this, the developed application is lightweight and focuses on reading and passing RFID tags' details.

Three major limitations have been found in this study. First, at the current stage, the nearest neighbor is only calculated based on signal strength. This can be improved if RF phase information is applied [15], [22]. Second, cases where the LO is inside drawers, which would decrease the signal strength, have not been tested. Third, an actual RFID smart phone was not used in the experiment, a powerful UHF Gen2 RFID tag reader connected to a laptop was used instead. This reader has a long reading range that is unlikely to be achieved with 
current RFID smart phones. However, with development of these technologies, this limitation should be solved.

\section{Conclusion}

This paper has introduced a novel RFID and ontology based solution to localize misplaced objects in home environments as well as designing and recommending the implementation of an entire HLSM system. The system generates the localization results in the form of user-friendly statements, such as "the glasses are at the kitchen table". The localization rule relies on the three nearest detailed navigation landmarks decided by RSSI. Experimental results show that this technique is promising.

\section{REFERENCES}

[1] R. Tesoriero, J. A. Gallud, M. D. Lozano, and V. M. R. Penichet, "Tracking autonomous entities using RFID technology," IEEE Transactions on Consumer Electronics, vol. 55, pp. 650-655, 2009.

[2] A. Ibrahim and D. Ibrahim, "Real-time GPS based outdoor WiFi localization system with map display," Advances in Engineering Software, vol. 41, pp. 1080-1086, 2010.

[3] R. Mautz, "The challenges of indoor environments and specification on some alternative positioning systems," in Proc. 6th Workshop on Positioning, Navigation and Communication, 2009, pp. 29-36.

[4] L. M. Ni, Y. Liu, Y. C. Lau, and A. P. Patil, "LANDMARC: indoor location sensing using active RFID," Wireless networks, vol. 10, pp. 701-710, 2004.

[5] D. Fortin-Simard, K. Bouchard, S. Gaboury, B. Bouchard, and A. Bouzouane, "Accurate passive RFID localization system for smart homes," in Proc. IEEE 3rd International Conference on Networked Embedded Systems for Every Application, 2012, pp. 1-8.

[6] J. Ziegler, M. Graube, and L. Urbas, "RFID as universal entry point to linked data clouds," in Proc. IEEE International Conference on RFIDTechnologies and Applications, 2012, pp. 281-286.

[7] Y. Song, B. Han, X. Zhang, and D. Yang, "Modeling and simulation of smart home scenarios based on Internet of Things," in Proc. 3rd IEEE International Conference on Network Infrastructure and Digital Content, 2012, pp. 596-600.

[8] H. Dae-Man and L. Jae-Hyun, "Design and implementation of smart home energy management systems based on zigbee," IEEE Transactions on Consumer Electronics, vol. 56, pp. 1417-1425, 2010.

[9] N. Fallah, I. Apostolopoulos, K. Bekris, and E. Folmer, "Indoor Human Navigation Systems: A Survey," Interacting with Computers, vol. 25, pp. 21-33, 2013.

[10] S. M. Huynh, D. Parry, A. Fong, and J. Tang, "Home localization system for misplaced objects," in Proc. IEEE International Conference on Consumer Electronics, 2014, pp. 462-463.

[11] R. Tesoriero, J. Gallud, M. Lozano, and V. Penichet, "Using active and passive RFID technology to support indoor location-aware systems," IEEE Transactions on Consumer Electronics, vol. 54, pp. 578-583, 2008.

[12] K. Domdouzis, B. Kumar, and C. Anumba, "Radio-Frequency Identification (RFID) applications: A brief introduction," Advanced Engineering Informatics, vol. 21, pp. 350-355, 2007.

[13] A. Almaaitah, K. Ali, H. S. Hassanein, and M. Ibnkahla, "3D passive tag localization schemes for indoor RFID applications," in Proc. IEEE International Conference on Communications, 2010, pp. 1-5.

[14] S. S. Saad and Z. S. Nakad, "A standalone RFID indoor positioning system using passive tags," IEEE Transactions on Industrial Electronics, vol. 58, pp. 1961-1970, 2011.
[15] L. Yuhong and Q. Ya, "An indoor localization of UHF RFID using a hybrid approach," in Proc. 2nd International Conference on Consumer Electronics, Communications and Networks, 2012, pp. 1653-1656.

[16] J. L. Brchan, L. Zhao, J. Wu, R. E. Williams, and L. C. Pérez, "A realtime RFID localization experiment using propagation models," in Proc. IEEE International Conference on RFID, 2012, pp. 141-148.

[17] E. DiGiampaolo and F. Martinelli, "A passive UHF-RFID system for the localization of an indoor autonomous vehicle," IEEE Transactions on Industrial Electronics, vol. 59, pp. 3961-3970, 2012.

[18] J. Han, Y. Zhao, Y. S. Cheng, T. L. Wong, and C. H. Wong, "Improving Accuracy for 3D RFID Localization," International Journal of Distributed Sensor Networks, vol. 2012, 2012.

[19] A. Athalye, V. Savic, M. Bolic, and P. M. Djuric, "A novel semi-passive rfid system for indoor localization," IEEE Sensors Journal, vol. 13, pp. 528-537, 2013

[20] R. Want, "An introduction to RFID technology," IEEE Pervasive Computing, vol. 5, pp. 25-33, 2006.

[21] P. Solic, Radic, x, J., and N. Rozic, "Software defined radio based implementation of RFID tag in next generation mobiles," IEEE Transactions on Consumer Electronics, vol. 58, pp. 1051-1055, 2012.

[22] P. V. Nikitin, R. Martinez, S. Ramamurthy, H. Leland, G. Spiess, and K. Rao, "Phase based spatial identification of UHF RFID tags," in Proc. IEEE International Conference on RFID, 2010, pp. 102-109.

[23] B. Motik, P. F. Patel-Schneider, and B. Parsia, "OWL 2 Web Ontology Language: Structural Specification and Functional-Style Syntax," W3C recommendation, vol. 27, p. 17, 2009.

\section{BIOGRAPHIES}

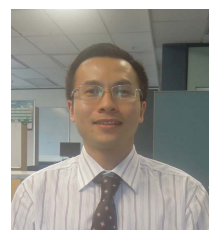

S. M. Huynh is currently undertaking $\mathrm{PhD}$ in Computing and Mathematical Sciences at Auckland University of Technology (AUT). He finished the Master of Computer and Information Sciences degree at AUT in 2014 after working as a software engineer, a team leader, and a project leader for TMA Solutions, CSC Vietnam, and Blueicon Technologies from 2005 to 2012. His research interests include fuzzy ontologies, Semantic Web, and RFID.

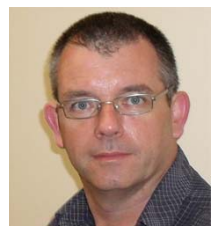

D. Parry (S'01-M'06) is an Associate Professor and director of the AUT Radio frequency Identification (RFID) laboratory (AURA) in the AUT School of Computer and Mathematical Sciences. His research interests include Health Informatics, Ontology based information retrieval, and RFID applications for pervasive computing.

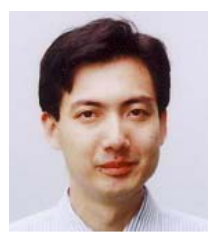

A. C. M. Fong (SM'04) is a Professor in the AUT School of Computer and Mathematical Sciences. He began his professional career in 1997 as an engineer with Motorola Corporate Research \& Technology Center - (later) Global Software Division, where he was engaged in the development of a range of consumer communication devices. His research interests include information processing and management, web-based systems, Semantic Web, and multimedia processing.

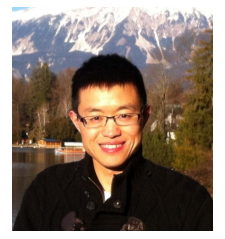

J. Tang (M'06-SM'14) is an Associate Professor in the Department of Computer Science and Technology, Tsinghua University. His main research interests include data mining algorithms and social network theories. He has been a visiting scholar at Cornell University, Chinese University of Hong Kong, Hong Kong University of Science and Technology, and Leuven University. 